\title{
MATHEMATICAL ANALYSIS OF TYPHOID INFECTION WITH TREATMENT
}

\section{MOATLHODI KGOSIMORE and GOSALAMANG R. KELATLHEGILE}

Botswana University of Agriculture and Natural Resources

Private Bag 0027

Gaborone

Botswana

e-mail: gkelatlhegile@bca.bw

\begin{abstract}
In this paper, we develop a deterministic model of typhoid that accounts for relapse of treatment. Mathematical analysis and numerical simulations are carried out to determine the transmission dynamics of typhoid in a community. The study aims to obtain insight on typhoid transmission dynamics and the role of carriers on the spread of the disease. Two equilibria exist: the disease-free equilibrium which is locally asymptotically stable if $R_{0}<1$ and unstable if $R_{0}>1$ and the endemic state which is stable if $R_{0}>1$. The simulation results suggest sustained epidemic in the long time. Implications of these results point to evolution of carriers due to relapse of treatment.
\end{abstract}

\section{Introduction}

Typhoid is a major public health concern in tropical developing countries, especially in areas where access to clean water and other sanitation measures are limited $[3,13,14]$. Typhoid fever has complex 2010 Mathematics Subject Classification: 92D30, 34D23.

Keywords and phrases: typhoid, treatment, transmission, reproduction number, diseasefree.

Received July 14, 2016

(C) 2016 Scientific Advances Publishers 
pathogenesis and manifests as an acute febrile disease, with relatively long incubation period that involves the transmigration of the microorganism through the Peyer's patch, localized multiplication in the mesenteric lymph nodes, and subsequent spread to the liver and spleen prior to showing clinical symptoms [17]. It is a serious life-threatening infection characterised by false diagnosis due to similar signs and symptoms with malaria, which leads to improper controls and management of the disease. Despite extensive work on typhoid, not much is understood on the biology of the human-adapted bacterial pathogen and the complexity of the disease in endemic areas, especially in Africa [18].

Globally, the burden of the disease is estimated at 21 million cases and 222000 deaths annually with high rates reported among children and adolescents in South and Eastern Asia and uncertain in Africa [5, 10, $16]$.

The symptoms are alleviated with antibiotic medications, however, a proportion of people treated for typhoid fever usually experience relapse, after a week of antibiotic treatment with symptoms which are milder and last for a shorter time compared with the original illness, requiring further treatment with antibiotics [2, 21]. Typhoid fever maybe prevented using vaccines, even though repeated mass vaccinations at intervals of 5 years interval may reduce the disease incidence, small gains re-observed at each subsequent vaccination [4]. The dynamics of typhoid fever involve multiple interactions between the human host, pathogen and environment, contributing to both direct human-to-human and indirect environment-to-human transmission pathways [7, 15]. Typhoid fever produces long-term asymptomatic carriers which play a pivotal role in the disease transmission.

In order to gain in-depth understanding of the complex dynamics of typhoid fever a number of studies have been conducted and published. Cvjetanovic et al. [4] constructed an epidemic model for typhoid fever in a stable population to study the transmission of infection at different levels of endemicity. Mushayabasa et al. [11] developed and analysed a 
deterministic mathematical model for assessment of the impact of treatment and educational campaigns on controlling typhoid out-break in Zimbabwe. Date et al. [5] reviewed various vaccination strategies using current typhoid vaccines to assess the rationale, acceptability, effectiveness, impact and implementation lessons in order to inform future public health typhoid control strategies. Watson and Edmunds [20] carried out an intensive review of typhoid fever transmission dynamics models and economic evaluation of vaccination. Clinicians, microbiologists, modellers, and epidemiologists worldwide need full understanding and knowledge of typhoid fever to effectively control and manage the disease [18].

In this paper, we developed a deterministic model that accounts for relapse after treatment leading to evolution of carriers. The study aims to investigate the role of carriers evolving from treatment relapse in the transmission dynamics of the disease.

\section{The Mathematical Model}

We formulate a mathematical model that describes the dynamics of typhoid infection in a population. The model subdivides the population $N$ into five (5) compartments depending on the epidemiological status of individuals. The compartments are susceptible $S$, infectives $I$, carriers $I_{c}$, treated infectives $T$, and the recovered $R$. We follow some existing studies $[1,11,14]$ which assume direct (human-to-human) transmission of typhoid infection, even though typhoid is largely transmitted indirectly (human-to-environment or environment-to-human) [13, 14]. The study further assumes that upon treatment, a fraction of individuals relapse, become carriers (primary reservoir of the disease) and contribute to the transmission dynamics of the disease. We adopt the mathematical model of carriers by Kalajdzievska and Li [9] and incorporate treatment and allow for relapse into the carriers. We assume that recovered individuals are permanently immune. The parameter $\gamma$ denotes the recovery rate while $\tau$ is the relapse rate. We further assume that carriers develop symptoms at a constant rate $\alpha$ and proceed to symptomatic infectives. 
The goal of the model is to investigate the effects of treatment in reducing the burden of typhoid infection and exacerbating the evolution of carriers.

The susceptible population is replenished by recruitment or births at a per capita rate $\Lambda$ and is decreased due to infection at a rate $B S$ and natural death at a constant rate $\mu$, where $B$ is the force of infection given by

$$
B=\frac{c \beta\left(I+\kappa_{1} I_{c}+\kappa_{2} T\right)}{N}
$$

and the modification parameters $k_{i} \mathrm{~s}$ accounting for infectiousness of individuals satisfy $k_{2} \leq 1 \leq k_{1}$. Thus, we assume that the rate of transmission of carriers is high as compared to other infectious individuals as they may be unaware of their disease status, followed by symptomatic infectives and consequently treated individuals. A susceptible individual may be infected via direct contact with infected, carriers or treated infectious individuals. A proportion $\rho$ of new infected individuals become carriers, while $1-\rho$ become symptomatic. Treated infective class gains individuals through treatment at constant rate $\sigma$ and is decreased due to recovery at a constant rate $\gamma$ and diseaseinduced deaths $\delta_{2}$. We further assume that there is no natural recovery from typhoid infection. The above description leads to the following system of ordinary differential equations:

$$
\begin{aligned}
\dot{S} & =\Lambda-B S-\mu S, \\
\dot{I} & =\rho B S+\alpha I_{c}-\left(\mu+\sigma+\delta_{1}\right) I, \\
\dot{I}_{c} & =(1-\rho) B S+\tau T-(\mu+\alpha) I_{c}, \\
\dot{T} & =\sigma I-\left(\mu+\gamma+\tau+\delta_{2}\right) T, \\
\dot{R} & =\gamma T-\mu R,
\end{aligned}
$$


where $N=S+I+I_{c}+T+R$ is the total population. Addition of all the equations of system (1), we obtain an equation governing changes in the total population

$$
\frac{d N}{d t}=\Lambda-\mu N-\delta_{1} I-\delta_{2} T \leq \Lambda-\mu N
$$

Integrating the differential inequality (2), we obtain

$$
\limsup _{t \rightarrow \infty} N(t) \leq \frac{\Lambda}{\mu} .
$$

This demonstrates that the model can be studied in the feasible region

$$
\Omega=\left\{\left(S, I, I_{c}, T, R\right) \in \mathbb{R}_{+}^{5}: N(t) \leq \frac{\Lambda}{\mu}\right\} .
$$

It is important to verify that all solutions of system (1) with non-negative initial conditions remain non-negative for all time $t$.

\section{Model Analysis}

\subsection{Positivity of solutions}

In this subsection, we employ the technique by Friedman and Lungu ([6]) to demonstrate that system (1) is positively invariant and wellposed. We consider system (1) in matrix form

$$
\left(\begin{array}{c}
\dot{S} \\
\dot{I} \\
\dot{I}_{c} \\
\dot{T} \\
\dot{R}
\end{array}\right)=\left(\begin{array}{rrrrc}
-\mu & 0 & 0 & 0 & 0 \\
0 & -\omega_{1} & \alpha I_{c} & 0 & 0 \\
0 & 0 & -\omega_{2} & \tau & 0 \\
0 & \sigma & 0 & -\omega_{3} & 0 \\
0 & 0 & 0 & \gamma & -\mu
\end{array}\right)\left(\begin{array}{c}
S \\
I \\
I_{c} \\
T \\
R
\end{array}\right)+\left(\begin{array}{c}
\Lambda-B S \\
\rho B S \\
(1-\rho) B S \\
0 \\
0
\end{array}\right)
$$

where $\omega_{1}=\mu+\sigma+\delta_{1}, \omega_{2}=\mu+\alpha$, and $\omega_{3}=\mu+\gamma+\tau+\delta_{2}$, which in compact form can be written as 


$$
\frac{d x_{i}}{d t}=f(x, t)=A_{i} x_{i}+H\left(x_{i}\right)
$$

for $x=\left(x_{1}, x_{2}, \cdots, x_{n}\right)^{\prime}$, where $x_{1}=S, x_{2}=I, x_{3}=I_{c}, x_{4}=T, x_{5}=R$ and $(.)^{\prime}$ denotes transpose. Then system (1) can be written as

$$
\frac{d x_{i}}{d t}=f(x, t)
$$

for $x=\left(x_{1}, x_{2}, \cdots, x_{n}\right)^{\prime}$. One can easily show that, system (3) satisfies the differential inequality

$$
\frac{d x_{i}}{d t} \geq A_{i} x_{i}+\sum_{j=1}^{n} C_{i j} x_{i}+\epsilon,
$$

for $i=1, \cdots, n$, with $C_{i j} \geq 0$ and $\epsilon>0$. If $x_{i}(0) \geq \epsilon$ for $i=1, \cdots, n$, then $x_{i}(t) \geq \epsilon$ for all $t \geq 0$.

Proof 3.1. Assume without loss of generality that $\epsilon>0$. The case $\epsilon=0$ is trivial through approximation of the system with a sequence $\epsilon=\epsilon_{k}$, which converges to zero as $k$ goes to infinity. Suppose now that $x_{i}(0) \geq \epsilon>0$, for $1 \leq i \leq n$ does not hold. Then there exists $t_{0}>0$ such that $x_{i}(t)>0$ for $1 \leq i \leq n, 0 \leq t<t_{0}$ and $x_{i}\left(t_{0}\right)=0$ for at least one $i$, say $i=i_{0}$. Then $x\left(i_{0}\right)$ is a decreasing function such that $\frac{d x_{i_{0}}}{d t}\left(t_{0}\right) \leq 0$.

From the differential inequality $\frac{d x_{i_{0}}}{d t} \geq A_{i} x_{i_{0}}+\sum_{j=1}^{n} C_{i j} x_{i_{0}}+\epsilon$, which is a contradiction. Thus, if $x_{i}(0) \geq \epsilon$ for $i=1, \cdots, n$, then $x_{i}(t) \geq 0$ for all $t \geq 0$.

\subsection{Steady state solutions}

To obtain the equilibrium points of system (1), we set the right-hand side of the system to zero. The model analysis yields two equilibrium points, namely, the disease-free equilibrium given by 


$$
E_{0}=\left(\frac{\Lambda}{\mu}, 0,0,0,0\right)
$$

and the endemic equilibrium,

$$
E=\left(S^{*}, I^{*}, I_{c}^{*}, T^{*}, R^{*}\right),
$$

whose coordinates in terms of the force of infection are

$$
\begin{aligned}
& S^{*}=\frac{\Lambda}{\mu+B^{*}}, \quad I^{*}=\frac{Q_{0} \Lambda B^{*}}{\mu+B^{*}}, \quad I_{c}^{*}=\frac{Q_{1} \Lambda B^{*}}{\mu+B^{*}}, \\
& T^{*}=\frac{Q_{2} \Lambda B^{*}}{\mu+B^{*}}, \quad R^{*}=\frac{Q_{3} \Lambda B^{*}}{\mu+B^{*}}, \quad B^{*}=Q\left(R_{0}-1\right),
\end{aligned}
$$

where

$$
\begin{aligned}
Q & =\frac{1}{\sum_{i=0}^{3} Q_{i}}, \\
Q_{0} & =\frac{\left(\mu+\gamma+\tau+\delta_{2}\right)(\alpha+\mu \rho)}{(\mu+\alpha)\left(\mu+\sigma+\delta_{1}\right)\left(\mu+\gamma+\tau+\delta_{2}\right)-\alpha \tau \sigma}, \\
Q_{1} & =\frac{(1-\rho)\left(\mu+\gamma+\tau+\delta_{2}\right)+\tau \sigma Q_{0}}{(\mu+\alpha)\left(\mu+\gamma+\tau+\delta_{2}\right)}, \\
Q_{2} & =\frac{\sigma Q_{0}}{\left(\mu+\gamma+\tau+\delta_{2}\right)}, \\
Q_{3} & =\frac{\gamma Q_{2}}{\mu}, \text { and } R_{0}=c \beta\left(Q_{0}+k_{1} Q_{1}+k_{2} Q_{2}\right) .
\end{aligned}
$$

\subsection{The basic reproduction number, $R_{0}$}

We compute the effective reproduction number of the model using the next generation operator method by van den Driescche and Watmough [19]. The effective reproduction number is defined as the average number of secondary infections generated by primary cases under a specific control (treatment in this case) strategy. Distinguishing new infections 
from other transitions in system (1), we obtain the two matrices $F$ and $V$ of generation of new infections and transition terms, respectively, expressed as

$$
F=\left(\begin{array}{ccc}
\rho c \beta & \rho k_{1} & \rho k_{2} c \beta \\
(1-\rho) c \beta & (1-\rho) k_{1} c \beta & (1-\rho) k_{2} c \beta \\
0 & 0 & 0
\end{array}\right)
$$

and

$$
V=\left(\begin{array}{ccc}
\mu+\sigma+\delta_{1} & -\alpha & 0 \\
0 & \mu+\alpha & -\tau \\
-\sigma & 0 & \mu+\gamma+\tau+\delta_{2}
\end{array}\right) \text {. }
$$

The effective reproduction number of the model is the dominant eigenvalue or spectral radius of the matrix $F V^{-1}$, thus

$$
R_{0}=Q_{0} \beta_{I}+Q_{1} \beta_{I_{c}}+Q_{2} \beta_{T}
$$

where $\beta_{I}=c \beta, \beta_{I_{c}}=k_{1} \beta_{I}$, and $\beta_{T}=k_{2} \beta_{I}$, so that

$$
R_{0}=\frac{c \beta\left[(\mu+\alpha)\left(\mu+\gamma+\tau+\delta_{2}\right)+k_{1} \sigma \tau\right]}{(\mu+\alpha)\left(\mu+\gamma+\tau+\delta_{2}\right)} Q_{0}+\frac{k_{2} \sigma c \beta}{\left(\mu+\gamma+\tau+\delta_{2}\right)} Q_{0}+\frac{k_{1}(1-\rho) c \beta}{(\mu+\alpha)}
$$

The threshold parameter $R_{0}$ can be re-written as $R_{0}=R_{0 I}+R_{0 I_{c}}+R_{0 T}$, where

$$
R_{0 I}=\frac{(\rho \mu+\alpha)\left(\mu+\gamma+\tau+\delta_{2}\right) c \beta}{(\mu+\alpha)\left(\mu+\sigma+\delta_{1}\right)\left(\mu+\gamma+\tau+\delta_{2}\right)-\alpha \tau \sigma}
$$

accounts for transmissions due to symptomatic infectives,

$$
R_{0 I_{c}}=\frac{k_{1} \sigma \tau c \beta Q_{0}}{(\mu+\alpha)\left(\mu+\gamma+\tau+\delta_{2}\right)}+\frac{k_{1}(1-\rho) c \beta}{(\mu+\alpha)}
$$


accounts for transmissions due to carriers, who may have acquired infection through direct transmission or treatment relapse transforming them into carriers; and the infections due to treated individuals is given by

$$
R_{0 T}=\frac{k_{2} \sigma Q_{0} c \beta}{\left(\mu+\gamma+\tau+\delta_{2}\right)} .
$$

\subsection{Stability results}

Linearisation of system (1) about the disease-free equilibrium leads to the Jacobian matrix

$$
J_{E 0}=\left(\begin{array}{ccccr}
-\mu & c \beta & k_{1} c \beta & k_{2} c \beta & 0 \\
0 & -W_{1}\left(1-\rho R_{I}\right) & W_{2} \hat{\alpha}+W_{2} \rho R_{I_{c}} & W_{3} \rho R_{T} & 0 \\
0 & W_{1}(1-\rho) R_{I} & -W_{2}\left(1-(1-\rho) R_{I c}\right) & (1-\rho) W_{3} R_{T} & 0 \\
0 & \sigma & 0 & -W_{3} & 0 \\
0 & 0 & 0 & \gamma & -\mu
\end{array}\right)
$$

The eigenvalues of $J_{E 0}$ are $\lambda_{1}=\lambda_{2}=-\mu$ and the other three are determined from the characteristic equation

$$
a_{3} \lambda^{3}+a_{2} \lambda^{2}+a_{1} \lambda+a_{0}=0
$$

where

$$
\begin{aligned}
& a_{3}=1, \\
& a_{2}=W_{1}(1-\rho) R_{I}+W_{2}\left(1-(1-\rho) R_{I_{c}}\right)+W_{3}, \\
& a_{1}=W_{1} W_{2}\left(1-\rho R_{I}\right)\left(1-(1-\rho) R_{I_{c}}\right)-W_{1}(1-\rho) R_{I}\left(W_{2} \hat{\alpha}+W_{2} \rho R_{I_{c}}\right), \\
& a_{0}=W_{1} W_{2} W_{3}\left(1-R_{0}\right),
\end{aligned}
$$

with $W_{1}=\mu+\sigma+\delta_{1}, W_{2}=\mu+\alpha, W_{3}=\mu+\gamma+\tau+\delta_{2}$ and

$$
R_{I}=\frac{c \beta}{W_{1}}, \quad R_{I_{c}}=\frac{k_{1} c \beta}{W_{2}}, \quad R_{T}=\frac{k_{2} c \beta}{W_{3}}, \quad \hat{\alpha}=\frac{\alpha}{W_{2}} .
$$


The quantity $R_{I}$ denotes the reproduction number of the model for a population consisting entirely of infected individuals, $R_{I c}$ represents the reproduction number of the model for a population consisting entirely of carrier individuals, while $R_{T}$ is the reproduction number of the model for a population consisting entirely of treated individuals. The following theorem summaries the locally stability results:

Theorem 3.2. The disease-free equilibrium (DFE) is locally asymptotically stable if $R_{0}<1$ and unstable otherwise.

\section{Numerical Simulations}

In this section, we carry out numerical simulations to illustrate analytical results of system (1), using data from published literature. We consider various treatment scenarios to investigate the effects of treatment in reducing the burden of the disease and evolution of carriers. The following parameter values extracted from literature shall be used for simulations (Table 1).

Table 1. Typhoid model parameter and their interpretations

\begin{tabular}{lccc}
\hline Description & Parameters & Value & Citation \\
\hline Recruitment rate & $\Lambda$ & $31.3-55 / 100$ & {$[14]$} \\
Per capital death rate & $\mu$ & $7.7-27.8 / 1000$ & {$[14]$} \\
Disease-induced deaths & $\delta_{1}, \delta_{2}$ & $0.03-0.02-0.001$ & {$[1,4,14]$} \\
Effective contacts & $c$ & 10 & {$[8]$} \\
Rate of transmission & $\beta$ & $0.000197 /$ day & {$[12]$} \\
Progression to symptomatic state & $\alpha$ & $1 / 90$ & {$[4]$} \\
Rate of recovery from treatment & $\gamma$ & $0.002485 /$ day & \\
New infections becoming carriers & $\rho$ & $0.003-0.80$ & {$[4,14]$} \\
Rate of treatment & $\sigma$ & $0.19-0.8$ & Varied \\
Proportion of treated individuals & $\tau$ & $1 / 18$ & {$[4]$} \\
Modification parameters & $k_{1}, k_{2}$ & $1-1.2$ & Varied \\
\hline
\end{tabular}


From the assumption $k_{2} \leq 1 \leq k_{1}$, we consider a hypothetical scenario in which the transmission coefficients $k_{1}$ and $k_{2}$, are related by a decreasing function of treatment $T$ given by $k_{1}=1+k_{2} e^{-\omega T}$. We want to address the question: how does treatment influence the number of carriers and eventual outcome of the epidemic? We explore this behaviour through variation of treatment rate $\sigma$.

Figures 1 and 2 show that increasing treatment has the effect of drastically increasing the prevalence of treated and untreated infectives to their maximum levels and steadily decreasing to their lowest levels before settling at endemic levels. These results demonstrate complexity in the dynamics of the disease transmission due to treatment effects. Therefore, there is need to understand the interaction between treatment, recovery, and relapse rates required to reduce the burden of the disease in order to develop effective intervention programs. The results show that exhibition of two peaks (Figure 3) occurring at early (maximum peak) and mid (minimum peak) stages of the epidemic. It is observed that high treatment rates lead to sustained endemic levels of carriers for a long time without reduction of the epidemic. 


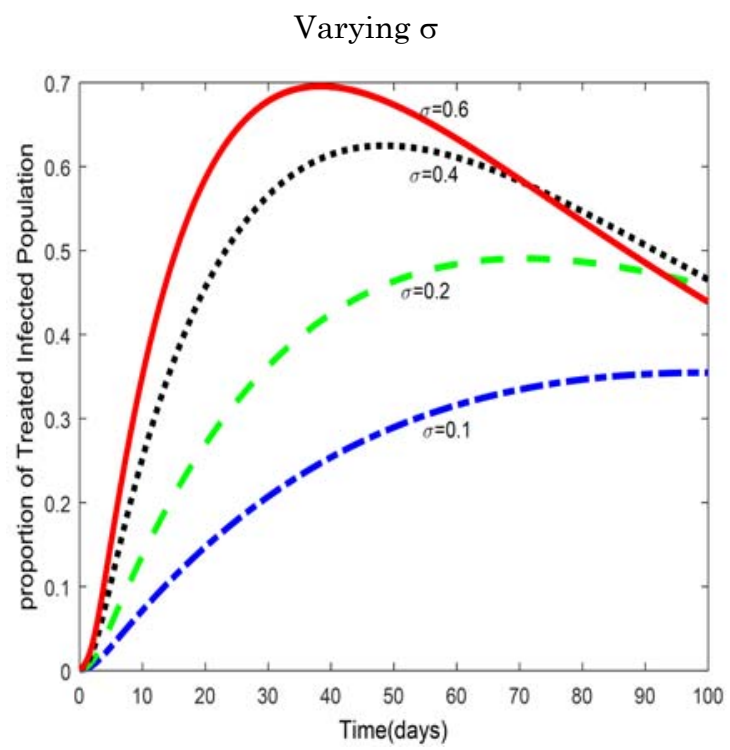

(a)

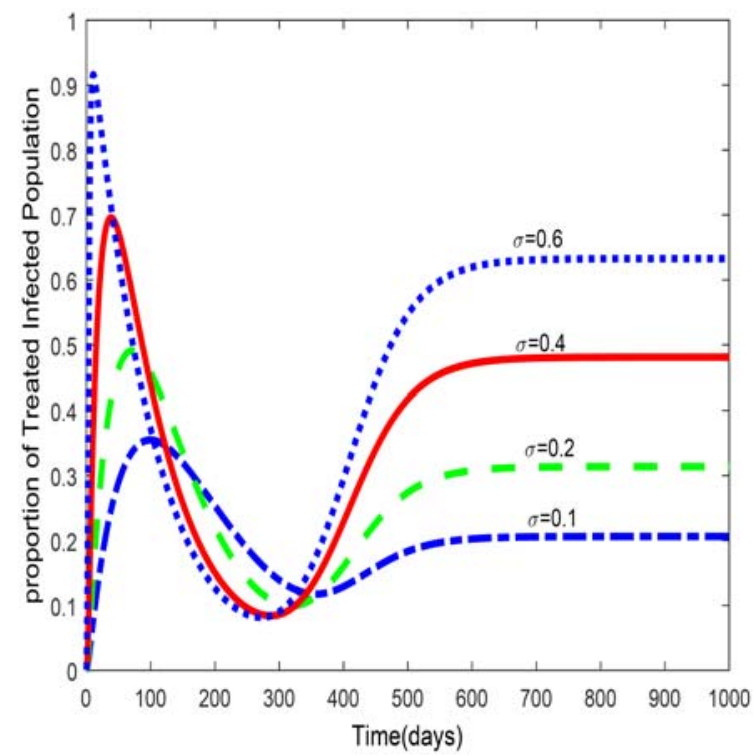

(b)

Figure 1. We vary $\sigma$ and fix: $\Lambda=55 / 1000, \delta_{1}=0.001, \delta_{2}=0.000247$, $\beta=0.125, \gamma=0.01, \rho=0.8, \mu=0.02, \alpha=0.45, \tau=0.056, \omega=0.4, e=2.718$, $k_{2}=1, k_{1}=1+k_{2} e^{-\omega T}$. 


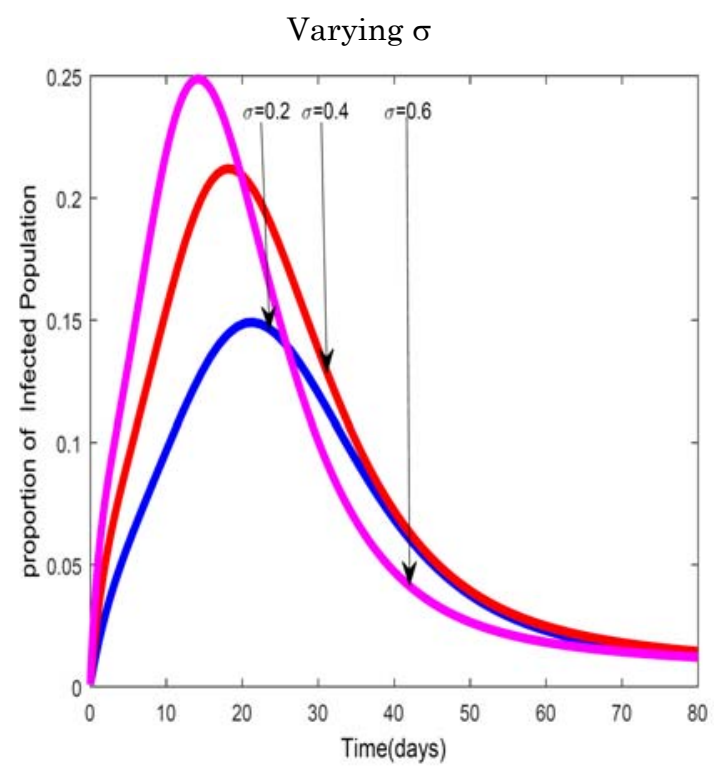

(a)

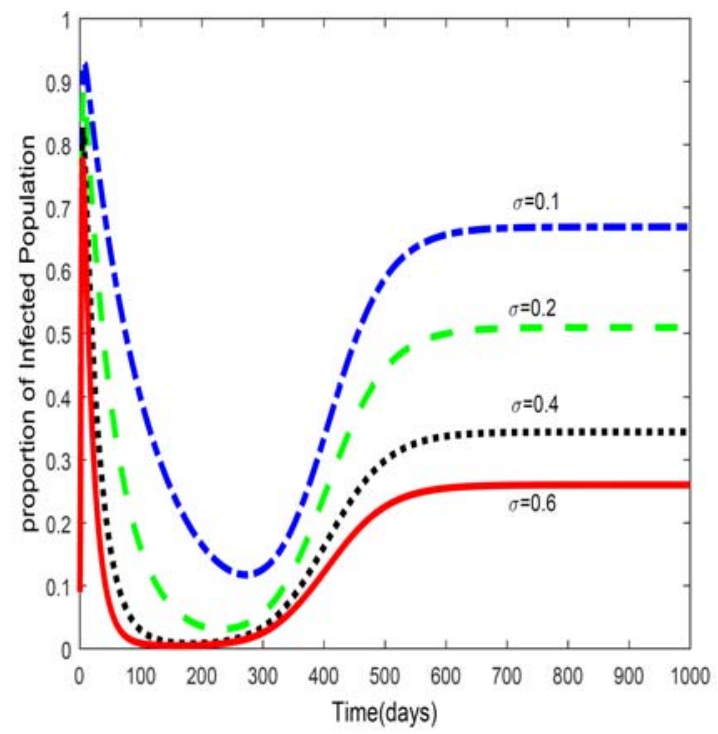

(b)

Figure 2. We vary $\sigma$ and fix: $\Lambda=55 / 1000, \delta_{1}=0.001, \delta_{2}=0.000247$, $\beta=0.125, \gamma=0.01, \rho=0.8, \mu=0.02, \alpha=0.45, \tau=0.056, \omega=0.4, e=2.718$, $k_{2}=1, k_{1}=1+k_{2} e^{-\omega T}$. 


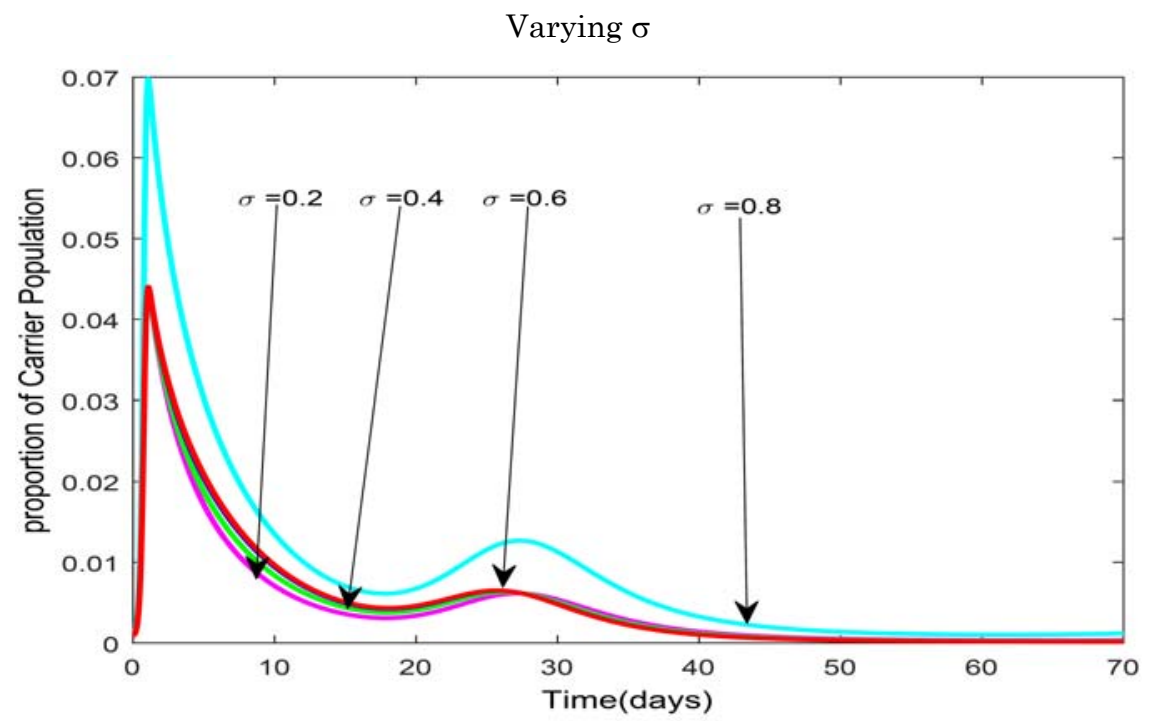

Figure 3. We vary $\sigma$ and fix: $\Lambda=55 / 1000, \delta_{1}=0.001, \delta_{2}=0.000247$, $\beta=0.125, \gamma=0.01, \rho=0.8, \mu=0.02, \alpha=0.45, \tau=0.056, \omega=0.4, e=2.718$, $k_{2}=1, k_{1}=1+k_{2} e^{-\omega T}$.

\section{Discussion}

In this study, we formulated and analyzed a deterministic typhoid infection model which incorporates treatment and assumes treatment relapse, leading to evolution of carriers. We established that the diseasefree equilibrium is locally asymptotically stable if $R_{0}<1$ and unstable if $R_{0}>1$. The endemic equilibrium exists and is stable if $R_{0}>1$. Numerical simulations suggested that increasing treatment sustains the typhoid epidemic in the population. Implications of this result points to an added effect from carriers evolving from treatment relapse. The dependence of modification transmission parameter $k_{2}$ on treated population provides insight in the role of treatment in the transmission dynamics of the disease. Due to complexity of the model closed form solutions of the population density dependent transmission rate could not 
be obtained. The study suggest development and implementation of preventive and treatment strategies which can reduce the burden of carriers in the population. Sensitive algorithms for case detection of infectives, especially carriers will play a critical role in reducing the burden of typhoid disease.

Epidemic trends guide allocation of resources, targeted design of control strategies and surveillance or improved techniques for data collection. Even though, this study provides insight on the transmission dynamics of typhoid infection, we ignored some of the current challenges associated with pathogenesis interaction and emergence of multi-drug resistance.

The dependence of infection parameters in the state variables seemed to suggest crucial dynamics appropriate to describe realistic behaviour of diseases.

\subsection{Conclusion}

We presented a deterministic model for typhoid transmission model with treatment. We determined conditions for existence and stability of equilibrium states characterized in terms of the effective reproduction number. The study showed that there is a disease-free equilibrium which is locally and globally asymptotically stable if $R_{0}<1$ and unstable if $R_{0}>1$, and the endemic equilibrium which is locally asymptotically stable if $R_{0}>1$. The study revealed further through simulations that the epidemic is sustained in the population. Implications of these results indicate that treatment sustain the carrier infectives who in turn sustains the epidemic in the population in the long run. Our paper is hypothetical and requires detailed study involving sensitivity analysis and parameter estimations to improve model predictions. 


\section{Acknowledgement}

We thank Professor C. J. Chikunji for his very helpful comments on the manuscript. The authors are also grateful to anonymous referees and the chief executive editor for their valuable comments, which constitute to the betterment of our manuscript.

\section{References}

[1] L. A. Adetunde, Mathematical methods for the dynamics of typhoid fever in Kassena-Nankana district of upper East region of Ghana, J. Mod. Math. Stat. 2(2) (2008), 45-49.

[2] B. Basnyat, The treatment of enteric fever, J. of the Royal Soc. of Med. 100 (2007), 161-162.

[3] J. A. Crump and E. D. Mintz, Global trends in typhoid and paratyphoid fever, Emerging Infections - CID 50 (2010), 241-246. DOI:10.1086/649541.

[4] B. Cvjetanovic, B. Grab and K. Uemura, Epidemiological model of typhoid fever and its use in the planning and evaluation of antityphoid immunization and sanitation programmes, Bull. Org. Mond. Sante (45) (1971), 53-75.

[5] K. A. Date, A. Bentsi-Enchill, F. Marks and K. Fox, Typhoid fever vaccination strategies, Vaccine 33 (2015), 55-61.

$$
\text { htt//dx.doi.org//10.101 6/j.vaccine.2015.04.028 }
$$

[6] A. Friedmann and E. Lungu, Can malaria parasite pathogenesis be prevented by treatment with tumor necrosis factor-alpha, Math. Biosci. Eng. 10 (2013), 609-624.

[7] J. Gonzalez-Guzman, An epidemiological model for direct and indirect transmission of typhoid fever, Maths. Biosci. 96 (1989), 33-46.

[8] M. Ghosh, P. Chandra, P. Sinha and J. B. Shukla, Modelling the spread of carrierdependent infectious diseases with environmental effect, Appl. Math. Comp. 152 (2004), 385-402.

[9] D. Kalajdzievska and M. Y. Li, Modelling the effects of carriers on transmission dynamics of infectious diseases, Mathematical Biosciences and Engineering 8(3) (2011), 711-722. doi:10.3934/mbe.2011.8.711.

[10] V. Mogasale, B. Maskery, R. L. Ochiai, J. S. Lee, V. V. Mogasale, E. Ramani, Y. E. Kim, J. K. Park and T. F. Wierzba, Burden of typhoid fever in low-income and middle-income countries: A systematic, literature-based update with risk-factor adjustment, Lancet Glob. Health 2 (2014), 570-580.

[11] S. Mushayabasa, C. P. Bhunu and N. A. Mhlanga, Modelling the transmission dynamics of typhoid in malaria endemic settings, Applicat. Appl. Math. Int. J. 6(1) (2014), 121-140. 
[12] S. Mushayabasa, Impact of vaccines on controlling typhoid fever in KassenaNankana district of upper East region of Ghana: Insights from mathematical model, J. Mod. Math. Stat. 5(2) (2011), 54-59.

[13] J. M. Mutua, B. Wang and N. K. Vaidya, Modelling malaria and typhoid fever co-infection dynamics, Mathematical Biosciences 264 (2015), 128-144.

[14] V. E. Pitzer, N. A. Feasey, C. Msefula, J. Mallewa, N. Kennedy, Q. Dube, B. Denis, M. A. Gordon and R. S. Heyerman, Mathematical modelling to assess the drivers of the recent emergence of typhoid fever in Blantyre, Malawi, CID 2015: 61(4) (2015), 251-258.

[15] V. E. Pitzer, C. C. Bowles, S. Baker, G. Kang, V. Balaji, J. J. Farrar and B. T. Grenfell, Predicting the impact of vaccination on the transmission dynamics of typhoid in South Asia: A mathematical modeling study, PLOS Negl. Trop. Dis. 8(1) (2014), e2642. doi:10.1371/journal.pntd.0002642.

[16] F. N. Qamar, A. Azmatullah and A. A. Bhutta, Challenges in measuring complications and death due to invassive Salmonella infections, Vaccine 33 (2015), 16-20.

[17] L. J. Thompson, S. J. Dunstan, C. Dolecek, T. Perkins, D. House, G. Dougan, T. H. Nguyen, T. P. Tran, C. D. Doan, T. P. Le, T. D. Nguyen, T. H. Tran, J. J. Farrar, D. Monack, D. J. Lynn, S. J. Popper and S. Falkow, Transcriptional response in the peripheral blood of patients infected with Salmonella enterica serovar Typhi, 106(52) (2009), 22433-22438.

www.pnas.org/cgi/doi/10.1073/pnas.0912386106

[18] J. Wain, R. S. Hendriksen, M. L. Mikoleit, K. H. Keddy and R. L. Ochia, Typhoid fever, The Lancet 385 (2015), 1136-1145.

http://dx.doi.org/10.1016/s0140-6736(13)62708-7

[19] P. van den Driessche and J. Watmough, Reproduction numbers and sub-threshold endemic equilibria for compartmental models of disease transmission, Math. Biosci. 180 (2002), 29-48.

[20] C. H. Watson and W. J. Edmunds, A review of typhoid fever transmission dynamic models and economic evaluations of vaccination, Vaccine 33 (2015), 42-54.

http://dx.doi.org/10.1016/j.vaccine.2015.04.013

[21] S. Zaki, Re-infection of typhoid fever and typhoid vaccine (comment on an imported enteric fever caused by a quinolone-resistant Salmonella typhi), Ann. Saudi Med. 31(2) (2011), 203-204. doi:10.4103/0256-4947.77505. 